OPEN ACCESS

Edited by:

Dongmei Deng,

VU University Amsterdam,

Netherlands

Reviewed by:

Mingyun $\mathrm{Li}$,

Sichuan University, China

Sayaka Katagiri,

Tokyo Medical and Dental University,

Japan

*Correspondence:

Zheng-Wei Huang

huangzhengwei@shsmu.edu.cn

Ying-Li Lu

luyingli2008@126.com

Rui Ma

marui1723@sina.com

${ }^{\dagger}$ These authors share first authorship

Specialty section:

This article was submitted to Microbiome in Health and Disease,

a section of the journal

Frontiers in Cellular

and Infection Microbiology

Received: 01 August 2020 Accepted: 05 November 2020 Published: 15 December 2020

Citation:

Zhao F, Dong T, Yuan K-Y, Wang N-J,

Xia F-Z, Liu D, Wang Z-M, Ma R, Lu Y-L and Huang Z-W (2020) Shifts in the Bacterial Community of

Supragingival Plaque Associated

With Metabolic-Associated

Fatty Liver Disease.

Front. Cell. Infect. Microbiol. 10:581888.

do: $10.3389 /$ fcimb.2020.581888

\section{Shifts in the Bacterial Community of Supragingival Plaque Associated With Metabolic-Associated Fatty Liver Disease}

\author{
Fen Zhao ${ }^{1,2,3 t}$, Ting Dong ${ }^{1,2,3 \dagger}$, Ke-Yong Yuan ${ }^{1,2,3}$, Ning-Jian Wang ${ }^{4}$, \\ Fang-Zhen Xia ${ }^{4}$, Di Liu ${ }^{5,6}$, Zhi-Min Wang ${ }^{7}$, Rui Ma ${ }^{1,2,3^{*}}$, Ying-Li Lu ${ }^{4 *}$ \\ and Zheng-Wei Huang ${ }^{1,2,3^{*}}$
}

1 Department of Endodontics, Shanghai Ninth People's Hospital, College of Stomatology, Shanghai Jiao Tong University School of Medicine, Shanghai, China, ${ }^{2}$ National Clinical Research Center for Oral Diseases, Shanghai, China, ${ }^{3}$ Shanghai Key Laboratory of Stomatology \& Shanghai Research Institute of Stomatology, Shanghai, China, ${ }^{4}$ Institute and Department of Endocrinology and Metabolism, Shanghai Ninth People's Hospital, Shanghai Jiao Tong University School of Medicine, Shanghai, China, ${ }^{5}$ Computational Virology Group, Center for Bacteria and Viruses Resources and Bioinformation, Wuhan Institute of Virology, Chinese Academy of Sciences, Wuhan, China, 6 University of Chinese Academy of Sciences, Beijing, China, ${ }^{7}$ Shanghai-MOST Key Laboratory of Health and Disease Genomics, Chinese National Human Genome Center, Shanghai, China

Metabolic-associated fatty liver disease (MAFLD), also known as the hepatic manifestation of metabolic disorders, has become one of the most common chronic liver diseases worldwide. The associations between some oral resident microbes and MAFLD have been described. However, changes to the oral microbial community in patients with MAFLD remain unknown. In this study, variations to the supragingival microbiota of MAFLD patients were identified. The microbial genetic profile of supragingival plaque samples from 24 MAFLD patients and 22 healthy participants were analyzed by 165 rDNA sequencing and bioinformatics analysis. Clinical variables, including indicators of insulin resistance, obesity, blood lipids, and hepatocellular damage, were evaluated with laboratory tests and physical examinations. The results showed that the diversity of the supragingival microbiota in MAFLD patients was significantly higher than that in healthy individuals. Weighted UniFrac principal coordinates analysis and partial least squares discriminant analysis showed that the samples from the MAFLD and control groups formed separate clusters (Adonis, $P=0.0120$ ). There were 27 taxa with differential distributions (linear discriminant analysis, LDA>2.0) between two groups, among which Actinomyces spp. and Prevotella 2 spp. were over-represented in the MAFLD group with highest LDA score, while Neisseria spp. and Bergeyella spp. were more abundant in the control group. Co-occurrence networks of the top 50 abundant genera in the two groups suggested that the inter-genera relationships were also altered in the supragingival plaque of MAFLD patients. In addition, in genus level, as risk factors for the development of MAFLD, insulin resistance was positively correlated with the abundances of Granulicatella, Veillonella, Streptococcus, and Scardovia, while obesity was positively correlated to the abundances of Streptococcus, Oslenella, Scardovia, and Selenomonas. Metagenomic 
predictions based on Phylogenetic Investigation of Communities by Reconstruction of Unobserved States revealed that pathways related to sugar (mainly free sugar) metabolism were enriched in the supragingival plaque of the MAFLD group. In conclusion, as compared to healthy individuals, component and interactional dysbioses were observed in the supragingival microbiota of the MAFLD group.

Keywords: metabolic-associated fatty liver disease, 16S rDNA sequencing, microbial community dysbiosis, insulin resistance, obesity, supragingival plaque

\section{INTRODUCTION}

Metabolic-associated fatty liver disease (MAFLD), formerly known as non-alcoholic fatty liver disease (NAFLD), refers to a wide spectrum of liver diseases characterized by the presence of hepatic steatosis in the absence of secondary causes, which include simple hepatic steatosis and steatohepatitis (Eslam et al., 2020). Progressive hepatic fibrosis is a hallmark of advanced steatohepatitis and can lead to cirrhosis, liver failure, and hepatocellular carcinoma (Sheka et al., 2020). MAFLD is an emerging public health concern worldwide, with a global pooled prevalence, by imaging, of $25.24 \%$ (Chalasani et al., 2018). The etiology of MAFLD is described as a complex hepatic manifestation of metabolic disorders, and the "multiple-hit" theory has been widely accepted as a potential mechanism involving insulin resistance (IR), obesity, chronic low grade inflammation, a sedentary lifestyle, regular consumption of a high fat diet, adipose tissue dysfunction, genetic factors, and gut microbial dysbiosis (Tiniakos et al., 2010; Buzzetti et al., 2016). With the increasing acknowledgement of the oral microbiome as a source of systemic inflammation, dysbiosis in the oral microbiome had been closely associated with metabolic diseases, including MAFLD (Acharya et al., 2017). A previous study found that the frequency of Porphyromonas gingivalis in the oral cavity is significantly higher in MAFLD patients as compared with health subjects (Yoneda et al., 2012). In another study, intravenous injection of sonicated $P$. gingivalis caused impaired glucose tolerance, IR, and liver steatosis in C57BL/6J mice fed a high-fat diet (Sasaki et al., 2018). However, because relatively few oral resident microbes have been the focus of previous studies, changes to the oral microbial community in patients with MAFLD remain unknown.

As a common site of oral sampling, the microbial profile of supragingival plaque is thought to reflect the health status of the host, such as gestation (Lin et al., 2018), type 2 diabetes (Artese et al., 2015), and other conditions (Espinoza et al., 2018). As compared with subgingival plaque, the supragingival microbiota can be acquired more easily and with less discomfort to the host (Lin et al., 2018). Therefore, sampling of the supragingival microbiota presents a promising method to assess oral and systematic health, especially for a large-scale health census. To the best of our knowledge, the present study is the first to assess the association between supragingival microbiota and MAFLD.

In the present study, supragingival plaques were obtained from 24 patients with MAFLD and 22 healthy controls. Illumina
MiSeq PE300 sequencing and bioinformatics analysis were employed to identify changes to the microbial profiles and inter-taxa relationships of the supragingival plaque of MAFLD patients. Specific microbial genera correlated with MAFLD and related clinical indexes were also identified. Furthermore, potential functional alterations to the supragingival microbiome were predicted based on the sequencing results. These findings would provide a deeper understanding of the oral ecological dysbiosis associated with MAFLD.

\section{MATERIALS AND METHODS}

\section{Study Population}

The study protocol was approved by the Ethics Committee of Shanghai Ninth People's Hospital affiliated with Shanghai Jiao Tong University, School of Medicine (Shanghai, China) (approval no. SH9H-2019-T295-1) and conducted in accordance with the tenets of the Declaration of Helsinki. Written informed consent was obtained from all participants prior to enrollment.

The study participants were recruited from a health census and assigned to one of two groups: the MAFLD group, consisting of 24 persons diagnosed with MAFLD via upper abdomen ultrasonography and other clinical examinations (Eslam et al., 2020), or a control group, consisting of 22 persons with normal findings by upper abdomen ultrasonography. Age and sex in the participants of the two groups were matched. For each participant, upper abdomen ultrasonography was performed successively by two experienced sonographers and those with the same diagnosis were enrolled.

The exclusion criteria were as follows: (i) the presence of other liver diseases (e.g., viral hepatitis, autoimmune hepatitis, and hepatolenticular degeneration); (ii) drug-induced hepatic steatosis (e.g., tamoxifen, amiodarone, valproate, methotrexate, and glucocorticoids); (iii) other factors that may cause hepatic steatosis (e.g., long-term total parenteral nutrition, inflammatory bowel disease, celiac disease, hypothyroidism, Cushing's syndrome, lipoprotein deficiency, lipid-atrophic diabetes, etc.); (iv) use of lipid-lowering drugs within the past 6 months; (v) type 1 or type 2 diabetes; (vi) current oral disease (e.g., untreated oral abscess, precancerous lesions, oral cancer, oral fungal infection, missing more than eight teeth, etc.); and (vii) other conditions (e.g., pregnant or lactating women, long-term heavy smoking, use of antibiotics for more than 5 days within the past 6 months, 
severe acute episode of a systematic disease, abnormal thyroid function, familial hyperlipidermia, etc.).

\section{Acquisition of Clinical Variables}

All participant demographics were retrieved from self-reported questionnaires and fasting blood samples were collected to detect clinical levels of total cholesterol (TC), total triglycerides (TG), low-density lipoprotein cholesterol (LDL-C), high-density lipoprotein cholesterol (HDL-C), alanine aminotransferase (ALT), aspartate aminotransferase (AST), gamma glutamyl transpeptidase (GGT), fasting plasma glucose (FPG), and fasting serum insulin (FSI). As an approximation of IR, the Homeostatic Model Assessment for Insulin Resistance (HOMAIR) equation was calculated as HOMA-IR $=$ FPG $(\mathrm{mmol} / \mathrm{L}) \times \mathrm{FSI}$ (mU/L)/22.5 (Matthews et al., 1985). The waist circumference, weight, height, and body mass index (BMI) of each participant were acquired via physical examination. The unpaired Student's t-test was applied for analysis of all clinical variables with an exception of "sex," which was analyzed using the Yates' continuity corrected chi-squared test.

\section{Dental Examination and Supragingival Plaque Collection}

An abbreviated dental exam was performed on all the participants by the same dentist using a periodontal probe. At least 9 teeth present and $>30 \%$ of the probed sites with attachment loss $\geq 1 \mathrm{~mm}$ were diagnosed as periodontitis (Armitage, 1999). The prevalence of periodontitis between the two groups was analyzed using the Yates' continuity corrected chi-squared test. Supragingival plaque was collected before eating in the morning in accordance with the methods described in the Manual of Procedures for the Human Microbiome Project (https://www.hmpdacc.org/hmp/doc/ HMP_MOP_Version12_0_072910.pdf) with minor modifications. The index teeth (\#3, \#9, \#12, \#19, \#25, and \#28) were isolated with cotton rolls and dried under a gentle stream of air. A sterile sickle scaler was used to collect the supragingival plaque from the buccal surfaces of the index teeth. Then, the scaler tips were immersed in $300 \mu \mathrm{l}$ of sterile normal saline contained in a sterile Eppendorf tube for 5-10 s with slight shaking and the surface of the scaler was wiped off on the inside edge of the tube. When the supragingival plaques of all index teeth were obtained, the Eppendorf tubes were sealed, marked, and kept frozen in liquid nitrogen until DNA extraction.

\section{DNA Extraction, Amplification, and High-Throughput Sequencing}

Total bacterial genomic DNA was extracted from the collected supragingival samples using the QIAamp DNA Mini Kit (Qiagen, Valencia, CA, USA) in accordance with manufacturer's protocols. The concentration and purification of the extracted DNA were determined using a NanoDrop 2000 UV-vis spectrophotometer (Thermo Scientific, Wilmington, DE, USA), while DNA quality was checked by $1 \%$ agarose gel electrophoresis.

The $16 \mathrm{~S}$ rDNA hypervariable $\mathrm{V} 3-\mathrm{V} 4$ region was PCRamplified with the forward primer 338F (5'-ACTCCTAC
GGGAGGCAGCAG-3') and the reverse primer 806R (5' GGACTACHVGGGTWTCT AAT-3') using a GeneAmp ${ }^{\text {TM }}$ PCR System 9700 (Applied Biosystems, Carlsbad, CA, USA). The parameters of the PCR reactions have been described in a previous study (Tong et al., 2019). Each PCR reaction was performed in triplicate and all resulting PCR products were extracted from $2 \%$ agarose gels and then further purified using the AxyPrep DNA Gel Extraction Kit (Axygen Biosciences, Union City, CA, USA) and quantified using a QuantiFluor ${ }^{\circledR}$ Single-Tube Fluorometer (Promega Corporation, Madison, WI, USA) in accordance with the manufacturer's instructions. Purified amplicons from different samples were pooled in equimolar concentrations and paired-end sequenced on an Illumina MiSeq PE300 sequencing platform (Illumina, Inc., San Diego, CA, USA).

\section{Data Processing and Bioinformatics Analysis}

Raw fastq files were quality-filtered using the Trimmomatic read trimming tool (https://kbase.us/) and merged using FLASH software (version 1.2.11; https://ccb.jhu.edu/software/FLASH/ index.shtml) in accordance with the criteria described in a previous report (Tong et al., 2019). After trimming, operational taxonomic units (OTUs) were clustered at a similarity cutoff value of $97 \%$ using the UPARSE algorithm (version 7.1; http://drive5.com/uparse/). The taxonomy of each $16 \mathrm{~S}$ rRNA gene sequence was analyzed with RDP Classifier algorithm (http://rdp.cme.msu.edu/) against the Silva 16S rRNA database (Release132 http://www.arb-silva.de), the confidence threshold was set to $70 \%$.

Alpha diversity indexes were calculated using MOTHUR software for describing and comparing microbial communities (version 1.30.2; https://www.mothur.org/wiki/Download_ mothur) and rarefaction curves were constructed at an intersequence similarity value of $97 \%$ using the QIIME bioinformatics pipeline (version 1.9.1; http://qiime.org/install/index.html). Bar plots were generated to visualize the species composition of all samples at the phylum and genus levels, and heat maps at the genus level were constructed using the R platform (version 3.6.1). Weighted UniFrac principal coordinates analysis (PCoA), nonparametric multivariate analysis of variance (Adonis), and partial least squares discriminant analysis (PLS-DA) were performed to identify differences in species composition between the MAFLD and control groups using QIIME. The linear discriminant analysis (LDA) effect size (LEfSe; http:// huttenhower.sph.harvard.edu/galaxy) was applied to identify the most discriminatory taxa between the groups at the phylum to genus levels. Taxa with logarithmic LDA scores of $>2.0$ were regarded as discriminative species. Co-occurrence networks of the 50 most abundant genera of each group were demonstrated using NetworkX (version 1.9.1). Spearman's correlation coefficients were calculated and those with a $|\rho|$ value of $>0.5$ and a probability $(P)$ value of $<0.05$ were visualized. Spearman's correlation coefficients among the clinical variables and the top 50 abundant genera of the supragingival microbiome were calculated, and the results were 
visualized as heat maps via the $\mathrm{R}$ platform. Furthermore, the bioinformatics software package Phylogenetic Investigation of Communities by Reconstruction of Unobserved States (PICRUSt2, version 1.1.0; http://picrust.github.io/picrust/) was used to predict the functional pathways of each group according to the Kyoto Encyclopedia of Genes and Genomes (KEGG). Statistical differences in top 30 abundant KEGG level 2 pathways and top 50 abundant KEGG level 3 pathways between two groups were determined by Wilcoxon rank-sum test with a Benjamini-Hochberg false discovery rate (FDR) correction to adjust $\mathrm{P}$ values for multiple testing.

\section{RESULTS}

\section{Subject Characteristics}

The demographic and clinical characteristics of the participants are summarized in Table 1. There were no significant differences in age, sex, blood pressure, heart rate and prevalence of periodontitis between the two groups. Subjects in the MAFLD group had relatively higher TG levels and lower HDL-C levels, and thus had higher TG/HDL-C ratios than the control group. Moreover, ALT and GGT levels were relatively higher in the MAFLD group than the control group. Because subjects with diabetes were excluded, there was no significant difference in FPG levels between the two groups, but FSI and HOMA-IR were significantly increased in the MAFLD group. Waist circumference

TABLE 1 | Demographic and clinical characteristics of the study participants.

\begin{tabular}{lccc}
\hline Parameter & Health(n=22) & MAFLD(n=24) & $\boldsymbol{P}$ \\
\hline Age (years) & $52.91 \pm 4.25$ & $52.75 \pm 4.39$ & 0.9013 \\
Sex (M: F) & $11: 11$ & $13: 11$ & 0.9898 \\
TC (mmol/L) & $5.77 \pm 0.78$ & $5.47 \pm 0.88$ & 0.2254 \\
TG (mmol/L) & $1.55 \pm 1.04$ & $2.51 \pm 1.28$ & $0.0083^{*}$ \\
LDL-C (mmol/L) & $3.41 \pm 0.52$ & $3.28 \pm 0.66$ & 0.4735 \\
HDL-C (mmol/L) & $1.40 \pm 0.36$ & $1.10 \pm 0.21$ & $0.0011^{*}$ \\
ALT (U/L) & $22.68 \pm 12.99$ & $40.25 \pm 27.55$ & $0.0092^{*}$ \\
AST (U/L) & $31.5 \pm 18.13$ & $38.21 \pm 19.09$ & 0.2292 \\
AST/ALT & $1.52 \pm 0.66$ & $1.11 \pm 0.43$ & $0.0142^{*}$ \\
GGT (U/L) & $24.50 \pm 21.16$ & $57.42 \pm 52.11$ & $0.0084^{*}$ \\
FPG (mmol/L) & $5.01 \pm 0.46$ & $5.18+0.56$ & 0.3196 \\
FSI(mU/L) & $4.02 \pm 2.68$ & $6.84 \pm 2.68$ & $0.0015^{\star}$ \\
HOMA-IR & $0.94 \pm 0.59$ & $1.58 \pm 0.66$ & $0.0013^{*}$ \\
Weight (kg) & $62.20 \pm 11.75$ & $68.23 \pm 9.64$ & 0.0631 \\
BMl & $24.13 \pm 3.00$ & $25.79 \pm 2.61$ & 0.0502 \\
WC(cm) & $81.84 \pm 9.45$ & $89.79 \pm 6.88$ & $0.0020^{*}$ \\
SBP (mm Hg) & $139.00 \pm 26.88$ & $136.46 \pm 19.65$ & 0.7144 \\
DBP (mm Hg) & $82.77 \pm 14.93$ & $82.79 \pm 12.17$ & 0.9962 \\
HR & $76.09 \pm 8.38$ & $73.71 \pm 10.25$ & 0.3954 \\
Periodontitis (\%) & 81.82 & 79.17 & 0.8843 \\
\hline Data are present & & & \\
\hline
\end{tabular}

Data are presented as the mean \pm standard deviation. ${ }^{*} P<0.05$. All clinical and demographic data were analyzed using the unpaired t-test with an exception of "sex" and "periodontitis", which were analyzed with the Yates' continuity corrected $\chi 2$ test. Abbreviations: ALT, alanine aminotransferase; AST, aspartate aminotransferase; BMI, body mass index; DBP, diastolic blood pressure; FPG, fasting plasma glucose; FSI, fasting serum insulin; GGT, gamma glutamyl trans-peptidase; HDL-C, high-density lipoprotein cholesterol; HOMA-IR, homeostatic model assessment of insulin resistance $[H O M A-I R=F P G(m m o l / L) \times F S I(m U / L) / 22.5] ; H R$, heart rate; LDL-C, low-density lipoprotein cholesterol; SBP, systolic blood pressure; TC, total cholesterol; TG, total triglycerides; WC, waist circumference. was significantly higher in the MAFLD group, illustrating visceral adiposity was ubiquitous in MAFLD patients.

\section{Bacterial Diversity and Community Structure of Supragingival Microbiota}

The concentration and purification of the extracted DNA met the requirements for further experiments (Table S1), results of $2 \%$ agarose gel electrophoresis revealed that the PCR products of the extracted DNA was qualified to further sequencing and analysis (Figure S1). A total of 2,318,404 high quality sequences were produced, with an average of 50,507 $\pm 11,212$ sequences per sample. In total, 23 phyla, 37 classes, 92 orders, 163 families, 339 genera, 628 species, and 1,021 OTUs were identified after taxonomic assignment of the sequences. All 16S rRNA gene sequences were submitted to the NCBI Sequence Read Archive (SRA) under bioproject accession PRJNA645880 (http://www.ncbi.nlm.nih.gov/sra).

As shown in Table 2, the MAFLD group had a higher Shannon index and lower Simpson index, suggesting the diversity of supragingival microbiota was higher in the MAFLD group than the control group (Table 2). Good's coverage indexes, which were close to 1 (Table 2), and rarefaction curves based on OTU levels (Figure S2) reached saturation plateaus, indicating that the sequencing depths were sufficient to represent the majority of the microbiota in both groups.

The taxa abundance of the supragingival microbiome from the two groups at the phylum and genus levels are depicted in Figures 1A, B, respectively. In general, the dominant taxa of the two communities were similar and consistent with the core species of the supragingival microbiome. The core phyla of all samples from both groups consisted of Bacteroidetes, Proteobacteria, Actinobacteria, Firmicutes, Fusobacteria, Patescibacteria, Epsilonbacteraeota, and Spirochaetes. Of the top 10 abundant genera, the MAFLD group had higher proportions of Capnocytophaga, Leptotrichia, Corynebacterium, Actinomyces, Streptococcus, Fusobacterium, Prevotella, and Veillonella; while Neisseria and Comamonas were more prevalent in the control group. The abundances of the top 50 abundant genera in each sample are displayed in the heat map presented in Figure S3.

Weighted UniFrac PCoA at the OTU level was employed to evaluate the similarity of the bacterial communities between the two groups. The results indicated that although samples from the two groups partly overlapped, there was a tendency of separation along the PC1 axis (Figure 1C). The results of

TABLE 2 | $\alpha$-diversity of supragingival microbiota in the MAFLD and Control groups.

\begin{tabular}{lccc}
\hline & MAFLD & Health & $\boldsymbol{P}$ \\
\hline Ace & $318.96 \pm 76.36$ & $285.74 \pm 82.16$ & 0.1561 \\
Chao 1 & $324.99 \pm 81.69$ & $287.67 \pm 77.17$ & 0.0969 \\
Shannon & $4.02 \pm 0.27$ & $3.80 \pm 0.39$ & $0.0303^{*}$ \\
Simpson & $0.04 \pm 0.01$ & $0.05 \pm 0.02$ & $0.0192^{*}$ \\
Coverage & $0.9983 \pm 0.0006$ & $0.9987 \pm 0.0006$ & 0.1319
\end{tabular}

Results are presented as the mean \pm standard deviation. ${ }^{*} P<0.05$. All $\alpha$-diversity estimators were analyzed using the Wilcoxon rank-sum test. 
A

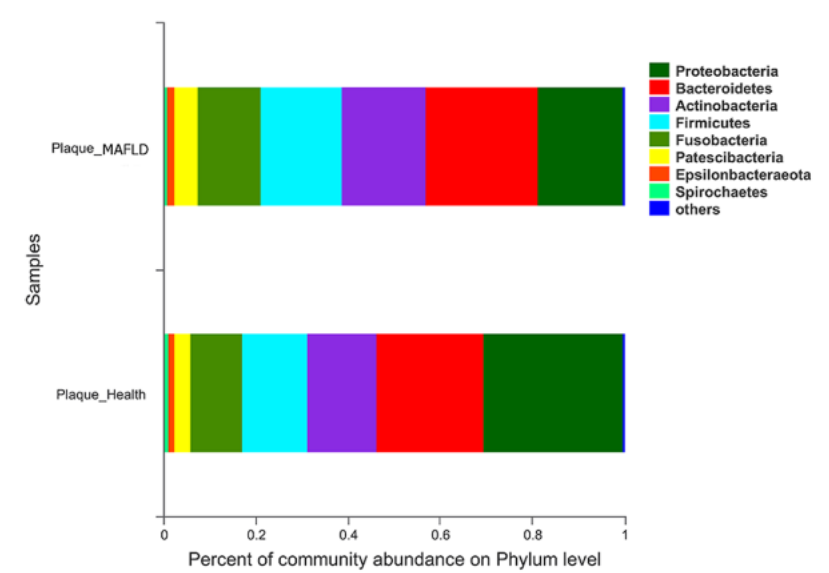

C

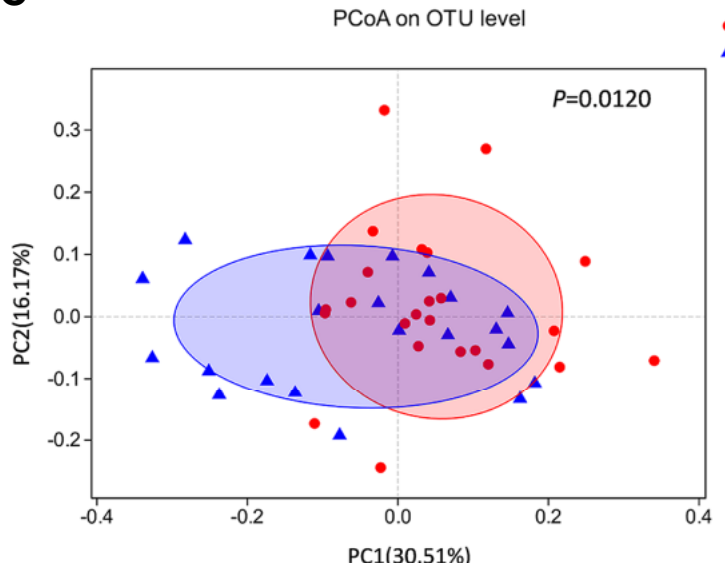

B

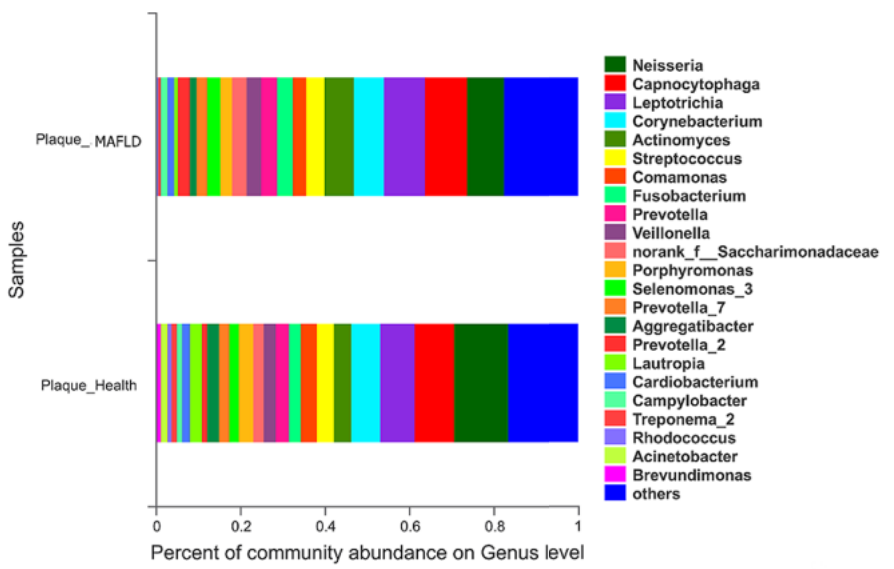

D - Paque_MAFLD

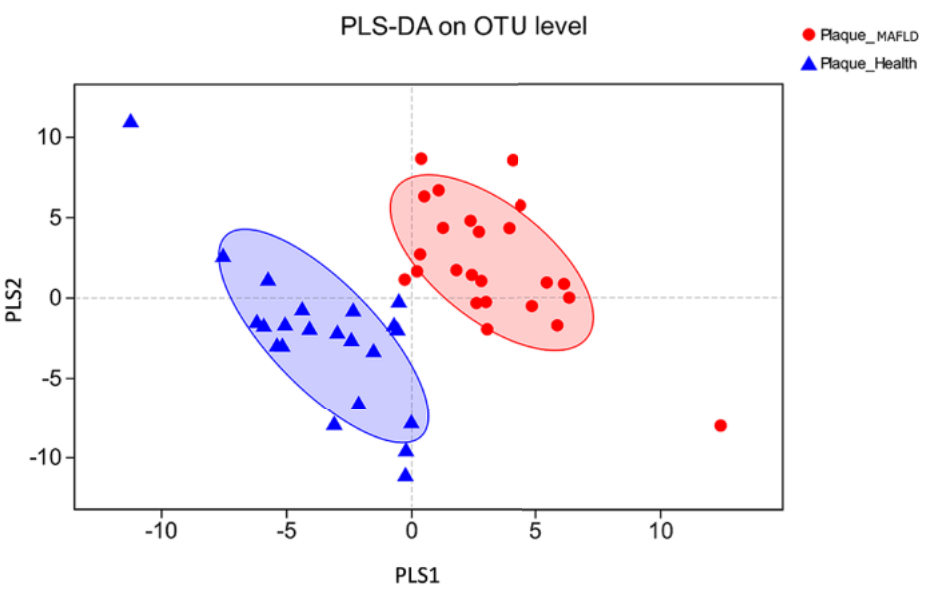

FIGURE 1 | Comparison of supragingival microbiota structures in the metabolic-associated fatty liver disease (MAFLD) and control groups. (A) Community structures at the phylum level. (B) Community structures at the genus level. (C) Weighted UniFrac principal coordinates analysis (PCOA) at the operational taxonomic unit (OTU) level. (D) Partial least squares discriminant analysis (PLS-DA) at the OTU level. 
Adonis $(P=0.0120)$ based on weighted UniFrac distances further verified the existence of significant differences in the overall structures of the supragingival microbiota of the two groups. PLS-DA, a supervised analysis suitable for high dimensional data, showed separate clustering of the samples from the MAFLD and control groups (Figure 1D), further demonstrating remarkable differences in the supragingival microbiota between the two groups.

\section{Alterations of the Supragingival Microbial Phylotypes/Inter-Genera Relationship Associated With MAFLD}

A circular cladogram based on the LEfSe results demonstrated differentially abundant taxa between the two groups (Figure 2A). Genera with logarithmic LDA scores of $>2.0(P<0.05)$ are plotted in Figure 2B and the selected taxa in other taxonomic level (from phylum to family) were shown in Figure S4. Briefly, there were 27 taxa with differential distributions between the two groups. At the genus level, Actinomyces, Prevotella 2, Scardovia, Megasphaera, and Alysiella were more abundant in the MAFLD group, while Neisseria, Bergeyella, Sphingomonas, and H1 were over-represented in the control group. Co-occurrence networks of the top 50 abundant genera of the two groups were depicted in Figures $3 \mathbf{A}, \mathbf{B}$. In general, the taxa within the main bacterial cluster ( $>5$ nodes and connected with intense lines) had stronger and more complex interrelationships in the MAFLD group.

\section{Correlations Between Clinical Variables and Supragingival Microbiota}

The heat map presented in Figure 4 depicts the correlations between the 50 most abundant bacterial genera and single clinical variables based on the Spearman's correlation coefficients. Of the top 50 abundant genera, HOMA-IR showed positive correlations with Streptococcus, Scardovia, Granulicatella, and Veillonella, but a negative correlation with Neisseria. Alloprevotella, and Peptostreptococcus were extremely significantly negatively correlated with TC and LDL-C levels. Aggregatibacter was negatively correlated with TG levels, but positively correlated with HDL-C levels. Streptococcus, Oslenella, Scardovia, and Selenomonas were significantly positively correlated with BMI and waist circumference. AST/ALT and GGT, two indicators of hepatocellular damage, were negatively correlated with Capnocytophaga.

\section{Predictive Metagenome Functional Profiling of the Supragingival Microbiomes of the MAFLD and Control Groups}

To detect functional differences in the supragingival microbiomes of the MAFLD and control groups, PICRUSt 2 was employed to predict the metagenome functional contents based on the $16 \mathrm{~S}$ rRNA datasets. Statistically significant differences $(P<0.05)$ in KEGG pathways were calculated with the Wilcoxon rank-sum test with FDR correction. As shown in Figure 5A, among the top 30 abundant KEGG level 2 pathways, Carbohydrate metabolism, Translation, Cellular communityprokaryotes and Membrane transport were significantly increased in the MAFLD group. At KEGG pathway level 3, starch and sucrose metabolism, fructose and mannose metabolism, and galactose metabolism were enriched in the MAFLD group. Meanwhile, a total of 9 pathways among the top 50 abundant KEGG level 3 pathways, including carbon metabolism, pyruvate metabolism, and the citrate cycle, were significantly decreased in the MAFLD group (Figure 5B).

\section{DISCUSSION}

The oral microbiota is attracting increased attention because of probable associations with metabolic disorders (Si et al., 2017; Chen et al., 2020; Wei et al., 2020). The associations between the oral microbiota and metabolic disorders can be explained in the following two aspects. On the one hand, microbial dysbiosis in the oral cavity is a source of systemic inflammation, which could lead to chronic low-grade inflammation and adversely affects the metabolic health of the host (Acharya et al., 2017). On the other hand, the oral microbiota can influence the composition of the gut microbiome, which plays important roles in metabolic health (Segata et al., 2012; Tremaroli and Bäckhed, 2012; Arimatsu et al., 2014; Li et al., 2019). Due to the anatomical position, about $10^{11}$ bacteria are swallowed from the oral cavity to the stomach every day (Segata et al., 2012), cultivation and sequencing techniques have also substantiated the association between the oral and gut microbiomes: Arimatsu et al. reported that oral administration of $P$. gingivalis significantly altered the Firmicutes/Bacteroidetes ratio, a significant index to evaluate the health status of the gut microbiome (Arimatsu et al., 2014); Li et al. found that the oral microbiota could overcome physical barriers and colonize the gut in gnotobiotic mice (Li et al., 2019). These findings acknowledged that the oral microbiota plays an important role in the development of metabolic diseases via "oral-gut axis". For MAFLD, although some oral resident microbes have been associated with the development of it (Yoneda et al., 2012; Sasaki et al., 2018), there has been no microbiome-wide association study of the association between the development of MAFLD and oral microbial ecology. Shaped by the health status of the host, supragingival plaque has been related to various metabolic disorders. For example, Hintao et al. reported significant differences in the microbial profiles of supragingival plaque between subjects with and without diabetes (Hintao et al., 2007); La Monte et al. found that metabolic syndrome was significantly associated with supragingival plaque (odds ratio $=1.74 ; 95 \%$ confidence interval $=1.22-2.50$ ) (LaMonte et al., 2014). Considering supragingival plaque can be obtained with minimal discomfort and risk (Lin et al., 2018), it was collected in this study to explore the ecological shifts of oral microbiota in MAFLD patients. By screening with strict inclusion/exclusion criteria and matching of confounding factors, the differences among the participants were minimized as much as possible in order to focus on compositional and structural differences of the supragingival microbiota in MAFLD patients.

The diversity of the supragingival microbiota of each group was determined using alpha diversity estimators. It is generally 
A

Plaque_MAFLD

Plaque_Health
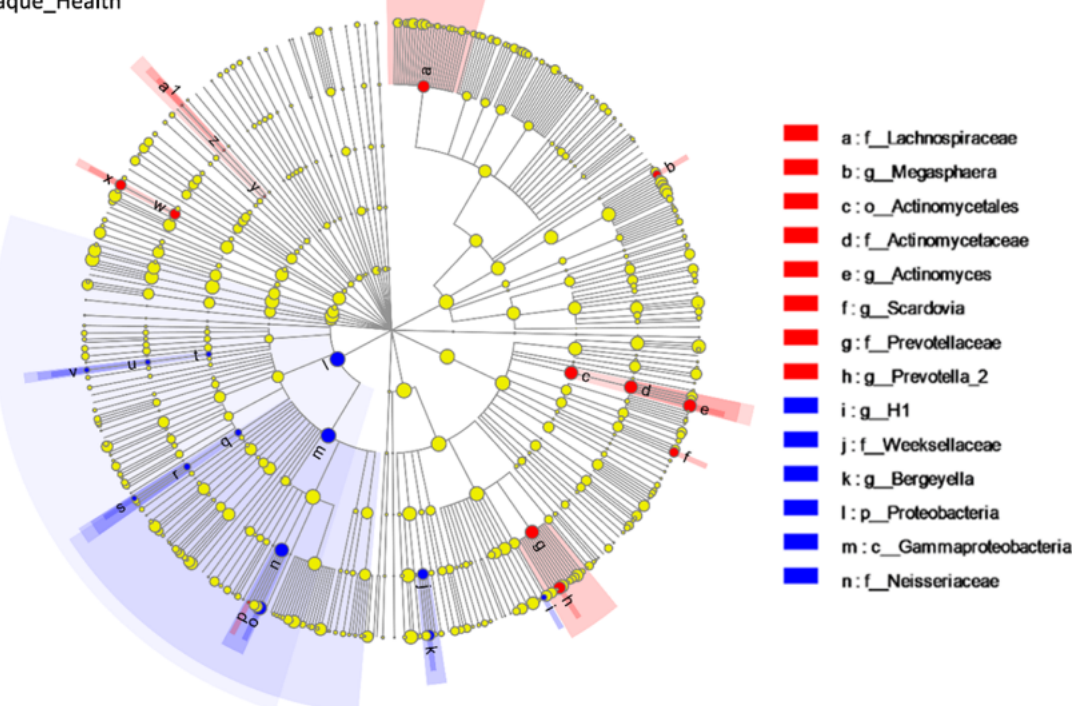

- o: : Neisseria

D: 2 _Aysiella

- q:0_Sphingomonadales

- rif_sphingomonadacea

- s:L_Sphingomonas

- t:o Bradymonadales

- u:__norank_o_Bradymonadales

- v: g_norank_f_norank_o_Bradymonadales

w:f_norank_o_Saccharimonadales

- $x:$ :_norank_f_norank_o_Saccharimonadales

- $y: 0$ : SBR 1031

- $2:$ :__norank_o_SBR1031

a1 :g_norankf_norank_o_SBR 1031

- n:f_Neisseriaceae

B Plaque_MAFLD

LEfSe Bar

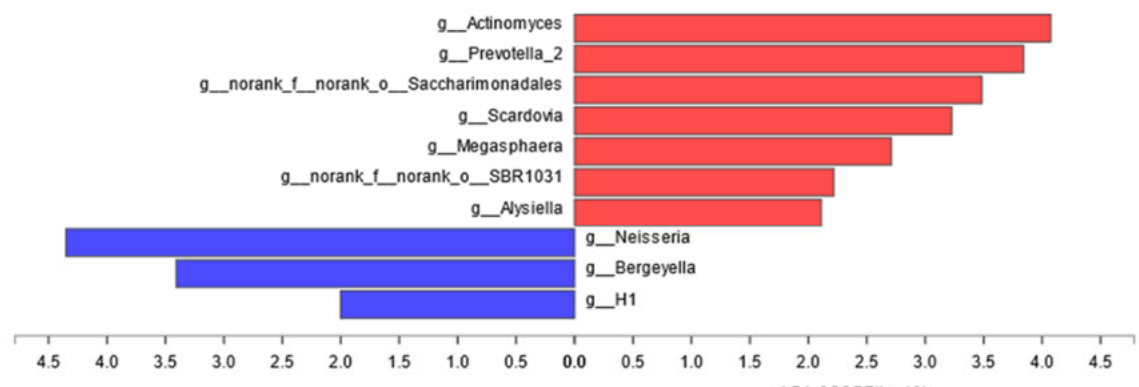

LDA SCORE(Iog10)

FIGURE 2 Bacterial phylotypes with altered abundances associated with MAFLD. (A) A cladogram of taxonomic representation based on LEfSe. Red indicates enrichment in samples from MAFLD patients and blue indicates the taxa enriched in samples from healthy controls. (B) A histogram of the logarithmic linear discriminant analysis (LDA) scores were calculated for the selected genera (logarithmic LDA $>2.0, P<0.05)$. 
A

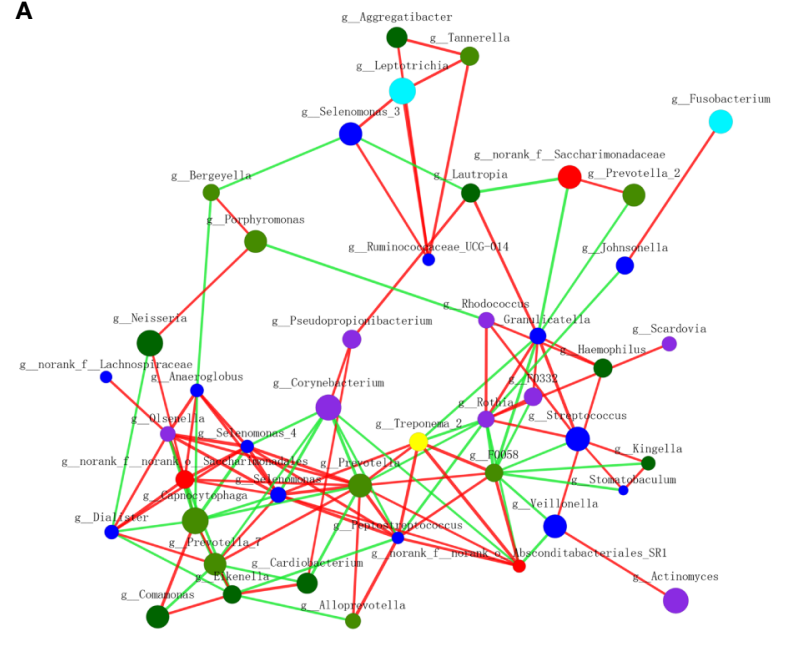

B

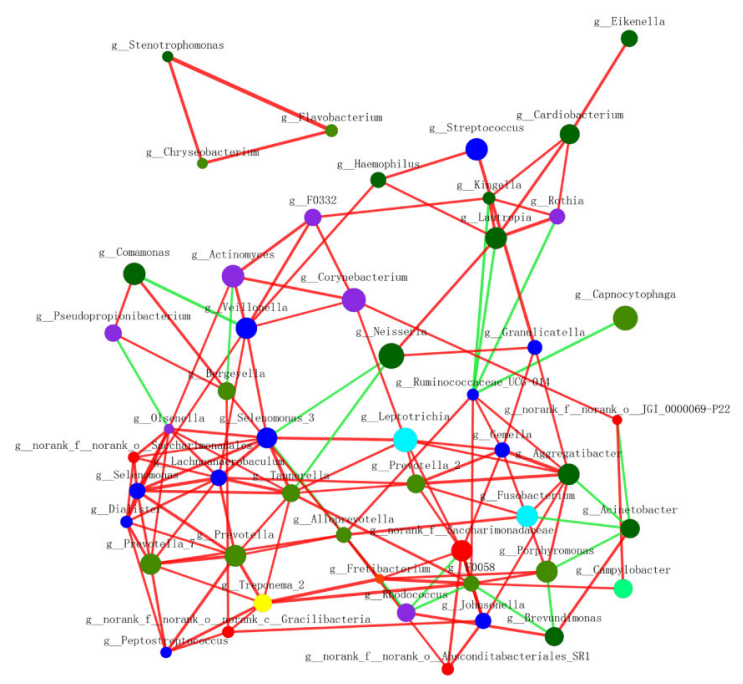

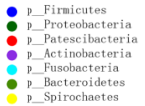
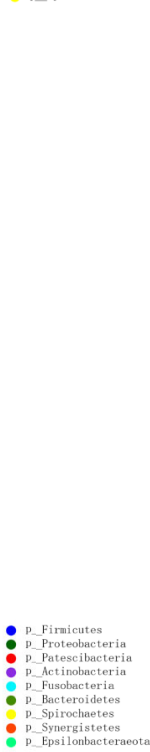

FIGURE 3 | Co-occurrence networks of the top 50 abundant genera in supragingival microbiota. The MAFLD group is shown in (A) and Health group in (B). The size of the node indicates the mean relative abundance of the corresponding genus. The same color represents genera belonging to the same phylum. The thickness of the connecting lines corresponds to the coefficient values $(P<0.05)$. The red and green lines indicate positive and negative correlations, respectively.

acknowledged that microbial diversity reflects the health status of the host. For example, decreased diversity of gut microbiota indicates functional or metabolic disorders in the host (Kelly et al., 2016), while increased diversity of oral microbiota is reported to imply poor oral (Camelo-Castillo et al., 2015; Takeshita et al., 2016) and holistic health (Armingohar et al., 2014; Si et al., 2017), because in a state of poor oral health, gingival bleeding provides a richer nutrient source (Segata et al., 2012). In the present study, increased diversity (lower Simpson index and higher Shannon index) of the supragingival microbiota in the MAFLD group was observed, suggesting possible alterations to the nutritional status of supragingival plaque in MAFLD patients. Consistent with previous studies (Utter et al., 2016; Lin et al., 2018), the core phyla identified in the present study included Proteobacteria, Bacteroidetes, Firmicutes, Actinobacteria, and
Fusobacteria, which accounted for $93.83 \%$ and $92.37 \%$ of the supragingival microbiomes of the control and MAFLD groups (Figure 1A), respectively. Similarly, although the proportions differed, the majority of the observed genera (including Capnocytophaga, Leptotrichia, Corynebacterium, Actinomyces, Streptococcus, Fusobacterium, Prevotella, Veillonella, Neisseria, and Comamonas) existed in both groups, thereby also supporting the core genera of the supragingival microbiota (Lin et al., 2018). A lower Firmicutes/Bacteroidetes ratio is considered as a healthy trait in both the oral cavity and gut (Chen et al., 2020). In the present study, the Firmicutes/Bacteroidetes ratio was lower in the supragingival plaque of the control group as compared to the MAFLD group (61.41\% vs. $72.38 \%$, calculated from Figure 1A respectively), indicating dysbiosis of the supragingival microbiota of the MAFLD group. 


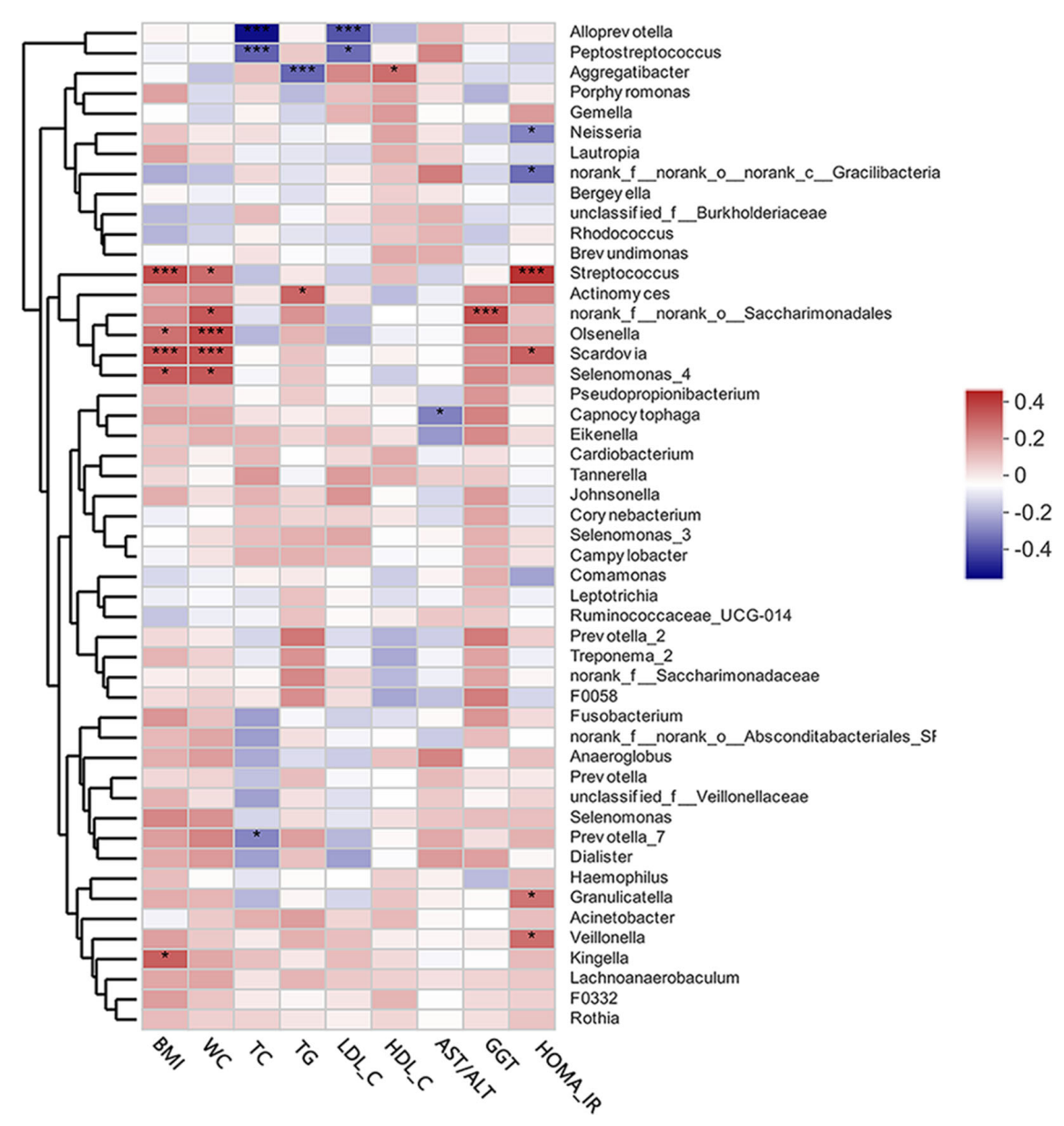

FIGURE 4 | A heat map of Spearman's correlation analysis of the top 50 abundant supragingival microbiota and clinical variables. The right side of the legend shows the color range of different $R$ values. Species clustering trees are presented on the left side of the heat map. ${ }^{*} P<0.05 ;{ }^{* \star} P<0.001$.

The PCoA and PLS-DA results demonstrated differences in the community compositions between the two groups (Adonis, $P=$ 0.0120). The discriminatory taxa between two groups were identified using LEfSe. At the genus level, Actinomyces and Prevotella 2 had the highest LDA scores in the MAFLD group. Actinomyces spp. is a normal resident bacteria of the oral cavity, which exerts important roles in biofilm formation (Polak et al., 2019). Actinomyces spp. has been associated with the severity of chronic periodontitis (Cao et al., 2018). Prevotella 2 is a genus of Gram-negative, anaerobic bacteria that exists in the gut and are relevant to multiple disease states, including an increased lifetime risk of cardiovascular disease (Kelly et al., 2016), ankylosing spondylitis (Chen et al., 2019), and increased levels of C-reactive protein (Sun et al., 2019). Considering the consistency between the oral and gut microbiotas (Segata et al., 2012; Arimatsu et al., 2014; Li et al., 2019), the prevalence of Prevotella 2 in the oral cavity is proposed as a potential marker of systematic diseases including MAFLD. In healthy participants, the genera Neisseria and Bergeyella had the highest LDA scores. Neisseria spp. is among the most abundant taxa in the oral cavity (Dong et al., 2018). A predominance of Neisseria spp. in the oral cavity indicates healthy conditions of the oral cavity (Meuric et al., 2017; Yamashita and Takeshita, 2017; Perez-Chaparro et al., 2018). Bergeyella spp. is a Gram-negative, aerobic bacteria (Muramatsu et al., 2019). In the present study, Bergeyella spp. was more prevalent in the control group, suggesting a negative correlation to MAFLD. Co-occurrence networks were used to predict inter-genera correlations of supragingival plaque between the two groups. As shown in Figure 3, there were significant differences in the interaction patterns of the two groups. In the MAFLD group, there were stronger and more complex interactions within the main cluster, but weaker correlations among the genera outside of the main cluster. Reportedly, an increase in interaction strength among taxa not only excludes other taxa, but decreases the stability of the microbial community (Ratzke et al., 2020). Therefore, it could be speculated that the supragingival microbial community of the MAFLD group was more unstable.

Inhibition of hepatic glucose production, increased accumulation of lipids in the liver, and IR are vital to the development of MAFLD (Bessone et al., 2019). It is currently 


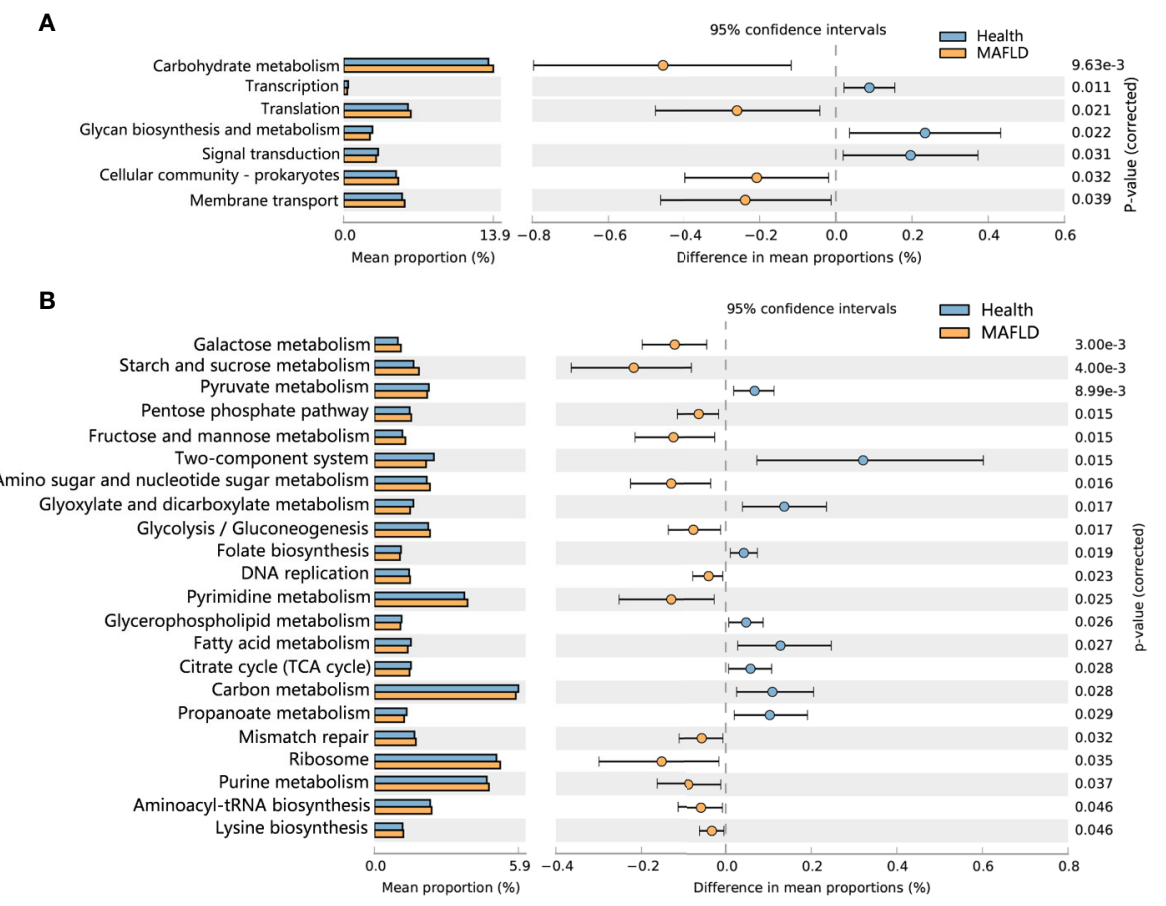

FIGURE 5 | Predictive metagenome functional profiling of supragingival microbiome of the MAFLD and control groups. (A) Relative abundance of predictive metagenome functional profiling of the top 20 abundant KEGG level 2 pathways (pathways with $P_{(\mathrm{FDR})}<0.05$ are shown). (B) Relative abundance of predictive metagenome functional profiling of the top 50 abundant KEGG level 3 pathways (pathways with $P_{(\text {FDR })}<0.05$ are shown).

believed that IR is an independent risk factor for the severity of MAFLD (Bessone et al., 2019). As first proposed by Matthews et al. in 1985, HOMA-IR is both practical and highly efficient for the evaluation of IR in both clinical and scientific studies (Matthews et al., 1985; Tang et al., 2015). In the present study, HOMA-IR was beyond the normal range (normal range $\leq 1$ ) in the MAFLD group and significantly higher than that in the control group $(P=0.0013)$ suggesting that IR is prevalent in patients with MAFLD. It was believed that chronic low-grade inflammation resulting from dysbiosis of the oral microbiota can reportedly aggravate IR (Bui et al., 2019). In this study, Spearman's correlation analysis revealed that the presence of Granulicatella spp., Veillonella spp., Streptococcus spp., and Scardovia spp. was positively correlated with HOMA-IR. It was reported that Granulicatella spp. has been positively correlated to periodontitis (Belstrøm et al., 2014), as well as infections outside of the oral cavity, such as infective endocarditis (Sasaki et al., 2020). Veillonella is a genus of Gram-negative anaerobic bacteria mainly found in the oral and gastrointestinal tracts. The presence of Veillonella spp. in the oral cavity has been correlated to increased production of pro-inflammatory cytokines (Bui et al., 2019; Li et al., 2020) and periodontal infections (Yamashita and Takeshita, 2017). Streptococcus spp. and Scardovia spp. are resident bacteria of the oral cavity that are closely related to caries formation (Kressirer et al., 2017). Although relatively few studies have investigated the relationship between caries-related bacteria and IR, patients with IR tend to have more decayed teeth (Loyola-Rodriguez et al., 2011).
In a state of chronic low-grade inflammation (Mervish et al., 2019), obesity is a contributor to various metabolic dysfunctions, such as MAFLD and type 2 diabetes (Canfora et al., 2019). As compared to BMI, visceral adiposity, as measured by waist circumference, has been closely linked to the severity of lipid deposition in the liver (Mervish et al., 2019), which is consistent with the results of the present study, which found an increase in waist circumference in MAFLD patients $(P=0.0020)$. In addition, multiple studies have verified the influence of obesity on the microbial profile of the oral cavity (Tam et al., 2018; Chattopadhyay et al., 2019). In this study, genera positively correlated with obesity mainly included Streptococcus, Oslenella, Scardovia, and Selenomonas. Streptococcus spp. and Scardovia spp. were also positively correlated to IR, supporting the positive association between obesity and IR (Mervish et al., 2019). The involvement of Oslenella spp. in endodontic infections (Vieira Colombo et al., 2016) and periodontal inflammation (Chen et al., 2020) have been well documented. In the Veillonellaceae family, Selenomonas is a genus of Gram-negative anaerobic bacteria. Members of Veillonellaceae family are considered to act as pro-inflammatory mediators (Tanner, 2015) and putative periodontal pathogens (Rocas and Siqueira, 2005). These results support the presumption that obesity is positively correlated to the abundance of bacteria associated with infectious diseases of the oral cavity (Maciel et al., 2016).

Dyslipidemia is a common clinical manifestation of MAFLD, especially hypertriglyceridemia and low serum HDL-C (Pacifico et al., 2014; Fukuda et al., 2016; Fan et al., 2019), which 
were also verified in this study $(P=0.0083$ for TG; $P=0.0011$ for HDL-C). Reportedly, oral infectious diseases and dyslipidemia could have a two-way relationship without a clear cause-andeffect relationship (Jaramillo et al., 2013). Actinomyces spp. has been positively correlated to TG levels as a potential indicator of MAFLD-related metabolic dysfunction. A surprising result was that the presence of Aggregatibacter spp. was negatively correlated with TG levels, but positively correlated with HDL-C levels, which might indicate good health, challenging the mainstream concept that the presence of Aggregatibacter spp. (especially A. actinomycetemcomitans) is related to dyslipidemia and other metabolic diseases (Jaramillo et al., 2013). Sampling sites may explain this discrepancy because Aggregatibacter spp. is a anaerobic bacteria with growth behaviors that may change in response to aerobic conditions (supragingival habitats). However, the exact reasons for this paradox remain unclear.

Known as indicators of hepatocellular damage, elevated serum levels of transaminases and transpeptidases are also main clinical manifestations of MAFLD (Sheka et al., 2020). Moreover, a decreased AST/ALT ratio is regarded as biomarker of progressive MAFLD (Sheka et al., 2020). In this study, a decreased AST/ALT ratio $(P=0.0142)$ as well as elevated GGT $(P=0.0084)$ were prevalent in MAFLD patients, suggesting the enrolled MAFLD patients had different degrees of hepatocellular damage. Capnocytophaga is a genus of Gram-negative anaerobic bacteria reportedly associated with periodontitis (Cao et al., 2018) and hyperglycemia (Graves et al., 2019). In this study, an abundance of Capnocytophaga spp. was negatively correlated to the AST/ALT ratio, suggesting it could be a potential biomarker of MAFLD progression.

Metagenomic predictions based on PICRUSt2 revealed that functional changes between the control and MAFLD groups mainly involved metabolism (Figure 5B). Among the KEGG pathways level 3, metabolism of sugars (mainly free sugars, including starch and sucrose, fructose and mannose, and galactose) were more prevalent in subjects with MAFLD, revealing that supragingival plaque in MAFLD patients can easily obtain nutrients, which could explain the increased microbial diversity observed in the supragingival plaque of the MAFLD group (Table 2). Pathways related to aerobic respiration (including pyruvate metabolism, and the citrate cycle) were more abundant in the supragingival plaque of the control group, suggesting that the proportion of aerobic bacteria in the supragingival plaque is higher in healthy people. However, a deficiency of predicting functions based on taxa composition is that bacterial functions can change with the health status of the host (Ikeda et al., 2020). Consequently, metatranscriptomics and metabolomics of the microbiota may provide more realistic functional profiles.

As this is a pilot study with matching of confounding factors, some intriguing findings surfaced, but still need to be verified in future studies with larger sample sizes. In addition, with the increasing attention to the functions of the oral microbial community, it is essential to identify changes to the actual functional profiles of the supragingival microbiota in MAFLD by metatranscriptomics and metabolomics.

\section{CONCLUSIONS}

In the present study, dysbiosis of supragingival microbiota associated with MAFLD was characterized. Briefly, the results revealed increases in the abundances of bacteria associated with oral infections, decreases in the abundances of potential beneficial aerobic bacteria, and changes in the interactions of the core microflora with the supragingival microbiota in patients with MAFLD. Moreover, as risk factors for the development of MAFLD, IR was positively correlated to the abundances of Granulicatella spp., Veillonella spp., Streptococcus spp., and Scardovia spp., while obesity was positively correlated with the abundances of Streptococcus spp., Oslenella spp., Scardovia spp., and Selenomonas spp. The increased free sugar metabolic pathways suggested that supragingival bacteria related to the metabolism of free sugars were associated with MAFLD. These findings provide a deeper understanding of the association between the oral microbiome and MAFLD, although further studies are needed to explore potential causal relationships.

\section{DATA AVAILABILITY STATEMENT}

The datasets presented in this study can be found in online repositories. The names of the repository/repositories and accession number(s) can be found below: https://www.ncbi. nlm.nih.gov/, PRJNA645880.

\section{ETHICS STATEMENT}

The studies involving human participants were reviewed and approved by the Ethics Committee of Shanghai Ninth People's Hospital affiliated with Shanghai Jiao Tong University, School of Medicine. The patients/participants provided their written informed consent to participate in this study.

\section{AUTHOR CONTRIBUTIONS}

FZ and TD performed the study design, data analysis, drafting, revising, final approval, and handled the accountability of all aspects of the work. K-YY and RM performed the bioinformatics analysis and data acquisition. N-JW and F-ZX performed the data analysis and acquisition. RM, Y-LL, DL, Z-MW, and Z-WH performed the study design, clinical sample collection, data analysis, revising, final approval and were also involved in the accountability of all aspects of the work. All authors contributed to the article and approved the submitted version.

\section{FUNDING}

This work was supported by grants from the Shanghai Top Priority Clinical Medicine Center (no. 2017ZZ01011), the Shanghai Municipal Key Clinical Specialty (no. shslczdzk1601), the Shanghai Clinical Research Center for Oral Diseases 
(no. 19MC1910600), the National Natural Science Foundation of China (no. 82071104/81570964/81371143), and partly supported by the Shanghai Ninth People's Hospital affiliated with Shanghai Jiao Tong University, School of Medicine (no. JYJC201806).

\section{ACKNOWLEDGMENTS}

We thank our coworkers for their assistance and all the study participants. We also thank Shanghai Majorbio Biopharm

\section{REFERENCES}

Acharya, C., Sahingur, S. E., and Bajaj, J. S. (2017). Microbiota, cirrhosis, and the emergingoral-gut-liver axis. JCI Insight 2 (19), e94416. doi: 10.1172/ jci.insight.94416

Arimatsu, K., Yamada, H., Miyazawa, H., Minagawa, T., Nakajima, M., Ryder, M. I., et al. (2014). Oral pathobiont induces systemic inflammationand metabolic changes associated with alteration of gut microbiota. Sci. Rep. 4, 4828. doi: $10.1038 /$ srep 04828

Armingohar, Z., Jorgensen, J. J., Kristoffersen, A. K., Abesha-Belay, E., and Olsen, I. (2014). Bacteria and bacterial DNA in atheroscleroticplaque and aneurysmal wall biopsies from patients with and without periodontitis. J. Oral. Microbiol. 6, 23408. doi: 10.3402/jom.v6.23408

Armitage, G. C. (1999). Development of a classification system for periodontal diseases and conditions. Ann. Periodontol. 4, 1-16. doi: 10.1902/annals.1999.4.1.1

Artese, H. P., Longo, P. L., Gomes, G. H., Mayer, M. P., and Romito, G. A. (2015). Supragingival biofilm control and systemicinflammation in patients with type 2 diabetes mellitus. Braz. Oral. Res. 29 (1), 1-7. doi: 10.1590/1807-3107BOR2015.vol29.0071

Belstrøm, D., Fiehn, N. E., Nielsen, C. H., Kirkby, N., Twetman, S., KlepacCeraj, V., et al. (2014). Differences in bacterial saliva profile between periodontitis patients and a control cohort. J. Clin. Periodontol. 41 (2), 104112. doi: $10.1111 /$ jcpe. 12190

Bessone, F., Razori, M. V., and Roma, M. G. (2019). Molecular pathways of nonalcoholic fatty liver disease development and progression. Cell Mol. Life Sci. 76 (1), 99-128. doi: 10.1007/s00018-018-2947-0

Bui, F. Q., Almeida-da-Silva, C. L. C., Huynh, B., Trinh, A., Liu, J., Woodward, J., et al. (2019). Association between periodontal pathogens and systemic disease. BioMed. J. 42 (1), 27-35. doi: 10.1016/j.bj.2018.12.001

Buzzetti, E., Pinzani, M., and Tsochatzis, E. A. (2016). The multiple-hit pathogenesis of non-alcoholic fatty liver disease (NAFLD). Metabolism 65 (8), 1038-1048. doi: 10.1016/j.metabol.2015.12.012

Camelo-Castillo, A. J., Mira, A., Pico, A., Nibali, L., Henderson, B., Donos, N., et al. (2015). Subgingival microbiota in health compared toperiodontitis and the influence of smoking. Front. Microbiol. 6, 119. doi: 10.3389/fmicb.2015.00119

Canfora, E. E., Meex, R. C. R., Venema, K., and Blaak, E. E. (2019). Gut microbial metabolites in obesity, NAFLD and T2DM. Nat. Rev. Endocrinol. 15 (5), 261273. doi: 10.1038/s41574-019-0156-Z

Cao, Y., Qiao, M., Tian, Z., Yu, Y., Xu, B., Lao, W., et al. (2018). Comparative Analyses of Subgingival Microbiome in Chronic Periodontitis Patients with and Without IgA Nephropathy by High Throughput $16 \mathrm{~S}$ rRNA Sequencing. Cell Physiol. Biochem. 47 (2), 774-783. doi: 10.1159/000490029

Chalasani, N., Younossi, Z., Lavine, J. E., Charlton, M., Cusi, K., Rinella, M., et al. (2018). The diagnosis and management of nonalcoholic fatty liver disease: Practice guidance from the American Association for the Study of Liver Diseases. Hepatology 67 (1), 328-357. doi: 10.1002/hep.29367

Chattopadhyay, I., Verma, M., and Panda, M. (2019). Role of Oral Microbiome Signatures in Diagnosisand Prognosis of Oral Cancer. Technol. Cancer Res. Treat 18, 1533033819867354. doi: 10.1177/1533033819867354

Chen, Z., Qi, J., Wei, Q., Zheng, X., Wu, X., Li, X., et al. (2019). Variations in gut microbial profiles in ankylosing spondylitis: disease phenotype-related dysbiosis. Ann. Transl. Med. 7 (20), 571. doi: 10.21037/atm.2019.09.41
Technology Co., Ltd. for providing the free online Majorbio Cloud Platform for the support provided for the bioinformatics analysis. This manuscript has been released as a pre-print at [ResearchGate] (Zhao et al., 2020).

\section{SUPPLEMENTARY MATERIAL}

The Supplementary Material for this article can be found online at: https://www.frontiersin.org/articles/10.3389/fcimb.2020. 581888/full\#supplementary-material

Chen, B., Wang, Z., Wang, J., Su, X., Yang, J., Zhang, Q., et al. (2020). The oral microbiome profile and biomarker inChinese type 2 diabetes mellitus patients. Endocrine 68 (3), 564-572. doi: 10.1007/s12020-020-02269-6

Dong, L., Yin, J., Zhao, J., Ma, S. R., Wang, H. R., Wang, M., et al. (2018). Microbial Similarity and Preference forSpecific Sites in Healthy Oral Cavity and Esophagus. Front. Microbiol. 9, 1603. doi: 10.3389/fmicb.2018.01603

Eslam, M., Newsome, P. N., Sarin, S. K., Anstee, Q. M., Targher, G., RomeroGomez, M., et al. (2020). A new definition for metabolic dysfunctionassociated fatty liver disease: An international expert consensus statement. J. Hepatol. 73 (1), 202-209. doi: 10.1016/j.jhep.2020.03.039

Espinoza, J. L., Harkins, D. M., Torralba, M., Gomez, A., Highlander, S. K., Jones, M. B., et al. (2018). Supragingival Plaque Microbiome Ecology andFunctional Potential in the Context of Health and Disease. mBio9 (6), e01631-18. doi: 10.1128/mBio.01631-18

Fan, N., Peng, L., Xia, Z., Zhang, L., Song, Z., Wang, Y., et al. (2019). Triglycerides to high-density lipoprotein cholesterol ratio as a surrogate for nonalcoholic fatty liver disease: a cross-sectional study. Lipids Health Dis. 18 (1), 39. doi: 10.1111/liv.12977

Fukuda, Y., Hashimoto, Y., Hamaguchi, M., Fukuda, T., Nakamura, N., Ohbora, A., et al. (2016). Triglycerides to high-density lipoproteincholesterol ratio is an independent predictor of incident fatty liver; a population-based cohort study. Liver Int. 36 (5), 713-720. doi: 10.1111/liv.12977

Graves, D. T., Correa, J. D., and Silva, T. A. (2019). The Oral Microbiota Is Modified by Systemic Diseases. J. Dent. Res. 98 (2), 148-156. doi: 10.1177/ 0022034518805739

Hintao, J., Teanpaisan, R., Chongsuvivatwong, V., Ratarasan, C., and Dahlen, G. (2007). The microbiological profiles of saliva, supragingival and subgingival plaque and dental caries in adults with and without type 2 diabetes mellitus. Oral. Microbiol. Immunol. 22 (3), 175-181. doi: 10.1111/j.1399-302X.2007. 00341.x

Ikeda, E., Shiba, T., Ikeda, Y., Suda, W., Nakasato, A., Takeuchi, Y., et al. (2020). Japanese subgingival microbiota in health vs disease and their roles in predicted functions associated with periodontitis. Odontology 108 (2), 280 291. doi: 10.1007/s10266-019-00452-4

Jaramillo, A., Lafaurie, G. I., Millán, L. V., Ardila, C. M., Duque, A., Novoa, C., et al. (2013). Association between periodontal disease and plasma levels of cholesterol and triglycerides. Colomb. Med. (Cali) 44 (2), 80-86. doi: 10.25100/ cm.v44i2.1123

Kelly, T. N., Bazzano, L. A., Ajami, N. J., He, H., Zhao, J., Petrosino, J. F., et al. (2016). Gut Microbiome Associates With Lifetime Cardiovascular Disease Risk Profile Among Bogalusa Heart Study Participants. Circ. Res. 119 (8), 956-964. doi: 10.1161/circresaha.116.309219

Kressirer, C. A., Smith, D. J., King, W. F., Dobeck, J. M., Starr, J. R., and Tanner, A. C. R. (2017). Scardovia wiggsiae and its potential role as a caries pathogen. J. Oral. Biosci. 59 (3), 135-141. doi: 10.1016/j.job.2017.05.002

LaMonte, M. J., Williams, A. M., Genco, R. J., Andrews, C. A., Hovey, K. M., Millen, A. E., et al. (2014). Association between metabolic syndrome and periodontal disease measures in postmenopausal women: the Buffalo OsteoPerio study. J. Periodontol. 85 (11), 1489-1501. doi: 10.1902/ jop.2014.140185

Li, B., Ge, Y., Cheng, L., Zeng, B., Yu, J., Peng, X., et al. (2019). Oral bacteria colonize and compete with gutmicrobiota in gnotobiotic mice. Int. J. Oral. Sci. 11 (1), 10. doi: 10.1038/s41368-018-0043-9 
Li, B. Z., Zhou, H. Y., Guo, B., Chen, W. J., Tao, J. H., Cao, N. W., et al. (2020). Dysbiosis of oral microbiota is associated withsystemic lupus erythematosus. Arch. Oral. Biol. 113, 104708. doi: 10.1016/j.archoralbio.2020.104708

Lin, W., Jiang, W., Hu, X., Gao, L., Ai, D., Pan, H., et al. (2018). Ecological Shifts of Supragingival Microbiotain Association with Pregnancy. Front. Cell. Infect. Microbiol. 8, 24. doi: 10.3389/fcimb.2018.00024

Loyola-Rodriguez, J. P., Villa-Chavez, C., Patiñio-Marin, N., Aradillas-Garcia, C., Gonzalez, C., and de la Cruz-Mendoza, E. (2011). Association between caries, obesity and insulin resistance in Mexican adolescents. J. Clin. Pediatr. Dent. 36 (1), 49-53. doi: 10.17796/jcpd.36.1.e25411r576362262

Maciel, S. S., Feres, M., Goncalves, T. E., Zimmermann, G. S., da Silva, H. D., Figueiredo, L. C., et al. (2016). Does obesity influence the subgingival microbiota composition in periodontal health and disease? J. Clin. Periodontol. 43 (12), 1003-1012. doi: 10.1111/jcpe.12634

Matthews, D. R., Hosker, J. P., Rudenski, A. S., Naylor, B. A., Treacher, D. F., and Turner, R. C. (1985). Homeostasis model assessment: insulin resistance and beta-cell function from fasting plasma glucose and insulin concentrations in man. Diabetologia 28 (7), 412-419. doi: 10.1007/bf00280883

Mervish, N. A., Hu, J., Hagan, L. A., Arora, M., Frau, C., Choi, J., et al. (2019). Associations of the Oral Microbiota withObesity and Menarche in Inner City Girls. J. Child Obes. 4 (1), 2. doi: 10.21767/2572-5394.100068

Meuric, V., Le Gall-David, S., Boyer, E., Acuna-Amador, L., Martin, B., Fong, S. B., et al. (2017). Signature of Microbial Dysbiosis inPeriodontitis. Appl. Environ. Microbiol. 83 (14), e00462-17. doi: 10.1128/aem.00462-17

Muramatsu, Y., Haraya, N., Horie, K., Uchida, L., Kooriyama, T., Suzuki, A., et al. (2019). Bergeyella zoohelcum isolated from oral cavities of therapy dogs. Zoonoses Public Health 66 (8), 936-942. doi: 10.1111/zph.12644

Pacifico, L., Bonci, E., Andreoli, G., Romaggioli, S., Di Miscio, R., Lombardo, C. V., et al. (2014). Association of serum triglyceride-to-HDL cholesterol ratio with carotid artery intima-media thickness, insulin resistance and nonalcoholic fatty liver disease in children and adolescents. Nutr. Metab. Cardiovasc. Dis. 24 (7), 737-743. doi: 10.1016/j.numecd.2014.01.010

Perez-Chaparro, P. J., McCulloch, J. A., Mamizuka, E. M., Moraes, A., Faveri, M., Figueiredo, L. C., et al. (2018). Do different probing depths exhibit striking differences in microbial profiles? J. Clin. Periodontol. 45 (1), 26-37. doi: $10.1111 /$ jcpe. 12811

Polak, D., Shany-Kdoshim, S., Zaydel, L., Feuerstein, O., and Houri-Haddad, Y. (2019). High-resolution novel method for tracking bacteria in a multi-species biofilm. Arch. Microbiol. 201 (2), 259-266. doi: 10.1007/s00203-018-1614-z

Ratzke, C., Barrere, J., and Gore, J. (2020). Strength of species interactions determines biodiversity and stability in microbial communities. Nat. Ecol. Evol. 4 (3), 376-383. doi: 10.1038/s41559-020-1099-4

Rocas, I. N., and Siqueira, J. F. Jr. (2005). Species-directed 16S rRNA gene nested PCR detection of Olsenella species in association with endodontic diseases. Lett. Appl. Microbiol. 41 (1), 12-16. doi: 10.1111/j.1472-765X.2005.01723.x

Sasaki, N., Katagiri, S., Komazaki, R., Watanabe, K., Maekawa, S., Shiba, T., et al. (2018). Endotoxemia by Porphyromonas gingivalisInjection Aggravates Nonalcoholic Fatty Liver Disease, Disrupts Glucose/Lipid Metabolism, andAlters Gut Microbiota in Mice. Front. Microbiol. 9, 2470. doi: 10.3389/fmicb.2018.02470

Sasaki, M., Shimoyama, Y., Ishikawa, T., Kodama, Y., Tajika, S., and Kimura, S. (2020). Contribution of different adherent properties of Granulicatella adiacens and Abiotrophia defectiva to their associations with oral colonization and the risk of infective endocarditis. J. Oral. Sci. 62 (1), 36-39. doi: 10.2334/josnusd.19-0021

Segata, N., Haake, S. K., Mannon, P., Lemon, K. P., Waldron, L., Gevers, D., et al. (2012). Composition of the adult digestive tract bacterial microbiome based on seven mouth surfaces, tonsils, throat and stool samples. Genome Biol. 13 (6), R42. doi: 10.1186/gb-2012-13-6-r42

Sheka, A. C., Adeyi, O., Thompson, J., Hameed, B., Crawford, P. A., and Ikramuddin, S. (2020). Nonalcoholic Steatohepatitis: A Review. JAMA 323 (12), 1175-1183. doi: 10.1001/jama.2020.2298
Si, J., Lee, C., and Ko, G. (2017). Oral Microbiota: Microbial Biomarkers of Metabolic Syndrome Independent of Host Genetic Factors. Front. Cell. Infect. Microbiol. 7, 516. doi: 10.3389/fcimb.2017.00516

Sun, Y., Chen, Q., Lin, P., Xu, R., He, D., Ji, W., et al. (2019). Characteristics of Gut Microbiota in PatientsWith Rheumatoid Arthritis in Shanghai, China. Front. Cell. Infect. Microbiol. 9, 369. doi: 10.3389/fcimb.2019.00369

Takeshita, T., Kageyama, S., Furuta, M., Tsuboi, H., Takeuchi, K., Shibata, Y., et al. (2016). Bacterial diversity in saliva and oralhealth-related conditions: the Hisayama Study. Sci. Rep. 6, 22164. doi: 10.1038/srep22164

Tam, J., Hoffmann, T., Fischer, S., Bornstein, S., Grassler, J., and Noack, B. (2018). Obesity alters composition and diversity of the oral microbiota in patients with type 2 diabetes mellitus independently of glycemic control. PloS One 13 (10), e0204724. doi: 10.1371/journal.pone.0204724

Tang, Q., Li, X., Song, P., and Xu, L. (2015). Optimal cut-off values for the homeostasis model assessment of insulin resistance (HOMA-IR) and prediabetes screening: Developments in research and prospects for the future. Drug Discov. Ther. 9 (6), 380-385. doi: 10.5582/ddt.2015.01207

Tanner, A. C. (2015). Anaerobic culture to detect periodontal and caries pathogens. J. Oral. Biosci. 57 (1), 18-26. doi: 10.1016/j.job.2014.08.001

Tiniakos, D. G., Vos, M. B., and Brunt, E. M. (2010). Nonalcoholic fatty liver disease: pathology and pathogenesis. Annu. Rev. Pathol. 5, 145-171. doi: 10.1146/annurev-pathol-121808-102132

Tong, Y., Zheng, L., Qing, P., Zhao, H., Li, Y., Su, L., et al. (2019). Oral Microbiota Perturbations Are Linked toHigh Risk for Rheumatoid Arthritis. Front. Cell. Infect. Microbiol. 9, 475. doi: 10.3389/fcimb.2019.00475

Tremaroli, V., and Bäckhed, F. (2012). Functional interactions between the gut microbiota and host metabolism. Nature 489 (7415), 242-249. doi: 10.1038/ nature 11552

Utter, D. R., Mark Welch, J. L., and Borisy, G. G. (2016). Individuality, Stability, and Variability of thePlaque Microbiome. Front. Microbiol. 7, 564. doi: $10.3389 /$ fmicb. 2016.00564

Vieira Colombo, A. P., Magalhaes, C. B., Hartenbach, F. A., Martins do Souto, R., and Maciel da Silva-Boghossian, C. (2016). Periodontal-disease-associated biofilm: A reservoir for pathogens of medical importance. Microb. Pathog. 94, 27-34. doi: 10.1016/j.micpath.2015.09.009

Wei, Y. S., Hsiao, Y. C., Su, G. W., Chang, Y. R., Lin, H. P., Wang, Y. S., et al. (2020). Identification of hyperglycemia-associatedmicrobiota alterations in saliva and gingival sulcus. Arch. Biochem. Biophys. 682, 108278. doi: 10.1016/j.abb.2020.108278

Yamashita, Y., and Takeshita, T. (2017). The oral microbiome and human health. J. Oral. Sci. 59 (2), 201-206. doi: 10.2334/josnusd.16-0856

Yoneda, M., Naka, S., Nakano, K., Wada, K., Endo, H., Mawatari, H., et al. (2012). Involvement of a periodontal pathogen, Porphyromonas gingivalis on the pathogenesis of non-alcoholic fatty liver disease. BMC Gastroenterol. 12, 16. doi: 10.1186/1471-230X-12-16

Zhao, F., Dong, T., Yuan, K.-Y., Xia, F.-Z., Liu, D., Wang, Z.-M., et al. (2020). Shifts in the bacterial community of supragingival plaque related to metabolic associated fatty liver disease. Preprint. doi: 10.21203/rs.3.rs-45886/v1

Conflict of Interest: The authors declare that the research was conducted in the absence of any commercial or financial relationships that could be construed as a potential conflict of interest.

Copyright (c) 2020 Zhao, Dong, Yuan, Wang, Xia, Liu, Wang, Ma, Lu and Huang. This is an open-access article distributed under the terms of the Creative Commons Attribution License (CC BY). The use, distribution or reproduction in other forums is permitted, provided the original author(s) and the copyright owner(s) are credited and that the original publication in this journal is cited, in accordance with accepted academic practice. No use, distribution or reproduction is permitted which does not comply with these terms. 